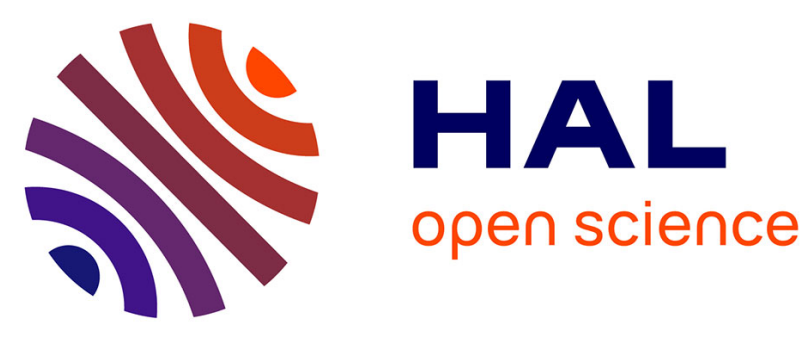

\title{
Endocrine and bone disease in appropriately treated adult patients with beta-thalassemia major
}

Marina Baldini, Stella Forti, Alessia Marcon, Fabio Massimo Ulivieri, Alessandra Orsatti, Benedetta Tampieri, Lorena Airaghi, Laura Zanaboni, Maria Domenica Cappellini

\section{To cite this version:}

Marina Baldini, Stella Forti, Alessia Marcon, Fabio Massimo Ulivieri, Alessandra Orsatti, et al.. Endocrine and bone disease in appropriately treated adult patients with beta-thalassemia major. Annals of Hematology, 2010, 89 (12), pp.1207-1213. 10.1007/s00277-010-1007-0 . hal-00550268

\section{HAL Id: hal-00550268 https://hal.science/hal-00550268}

Submitted on 26 Dec 2010

HAL is a multi-disciplinary open access archive for the deposit and dissemination of scientific research documents, whether they are published or not. The documents may come from teaching and research institutions in France or abroad, or from public or private research centers.
L'archive ouverte pluridisciplinaire HAL, est destinée au dépôt et à la diffusion de documents scientifiques de niveau recherche, publiés ou non, émanant des établissements d'enseignement et de recherche français ou étrangers, des laboratoires publics ou privés. 


\section{Editorial Manager(tm) for Annals of Hematology Manuscript Draft}

\section{Manuscript Number: AOHE-D-10-00052R1}

Title: Endocrine and bone disease in appropriately treated adult patients with beta-thalassemia major

Article Type: Original Article

Keywords: Thalassemia, osteoporosis, iron overload, endocrinopathy.

Corresponding Author: Dr marina baldini, MD

Corresponding Author's Institution: Internal Medicine $1 \mathrm{~A}$

First Author: Marina Baldini, MD

Order of Authors: Marina Baldini, MD; Stella Forti; Alessia Marcon, MD; Fabio Massimo Ulivieri, MD; Alessandra Orsatti, MSc; Benedetta Tampieri, MD; Lorena Airaghi, MD; Laura Zanaboni, MD; Maria Domenica Cappellini, PhD

Abstract: The natural history of beta thalassemia major (TM) has significantly changed during the last 2 decades. At present TM patients survive over their thirties and forties, but they have considerable morbidity. Bone demineralization is an important cause of morbidity in older patients; the etiology is multifactorial and partially unknown. We examined cross-sectionally 111 adult TM patients (66 females and 45 males, $32.6 \pm 6$ years), regularly transfused, properly chelated and replaced for endocrine defects. Bone demineralization was detected in $92.7 \%$ of patients, with different severity according to gender and site: osteopenia was the prominent finding at the femur, osteoporosis at lumbar spine $(\mathrm{p}<0.001)$, more evident in males. The femoral site was more influenced by biochemical and clinical factors; despite adequate replacement, femoral T-score was lower in the hypogonadic than in the eugonadic group ( $p=0.047)$. A significant correlation was found between bone mass and body mass index (BMI), alkaline phosphatase (ALP) and pre-transfusional Hb levels. Multivariate analysis indicated as significant regressors ALP, BMI and hypoparathyroidism (T-score: $\mathrm{p}=0.005,0.035,0.002$; Z-score: 002, 0.009, 0.003, respectively) at femur, only ALP at lumbar spine ( $\mathrm{p}=0.008$ and 0.045 for T and Z scores, respectively). Statistical significance was reached more frequently by T-score, while Zscore demonstrated to have a lower sensitivity.

Despite best care facilities, bone demineralization in thalassemic patients remains a challenge; further exploration of the relationships between bone loss and endocrine, biochemical and hematologic variables is warranted to find effective measures to reduce bone pain and fracture risk.

Response to Reviewers: We are grateful for the useful suggestions of the reviewers. We have revised our paper following their recommendations.

Reviewers' comments:

1) In the Discussion session they comment that "...the correlation between alkaline phosphatase and bone mineralization indicates a prevalent role of reduced deposition over increased reabsorption, as reported by other Authors, at variance with others. We could not confirm the same relationship with osteocalcin, another marker of osteoblastic activity.". 
First of all the authors have not measured any of the markers of bone resorption. I believe that it is important for the paper and for the the discussion to include at least NTX or CTX measurements. I hope that they have available serum for such measurement. I suggest that CTX is more sensitive than NTX in the serum.

- In the original paper, we did not include any marker of bone resorption because we had only partial data (different markers, incomplete series). It seems now evident that the importance of these indices to our work has been understated. In effect, we had available serum of most patients, so we decided to measure CTX following the useful suggestion of the reviewer; we recognize that this adjunct increases the scientific interest of our results.

As pointed out in the revised paper (page 8, lines 1 to 3) "no significant differences were found in CTX serum levels between thalassemic patients and controls, nor significant correlations with any of the studied parameters. This result may be due to the wide variability of the values in both cohorts; the problem should probably be obviated by a longitudinal study protocol."

2) Second, in order to state that the correlation of bALP with BMD indicates a prevalent role of reduced deposition, someone could expect a strong correlation. On the contrary the correlation was weak $(\mathrm{r}<-$ 0.25). Furthermore, we do not know if the bALP values were reduced (that will indicate reduced formation) or not.

- The correlation was found between total ALP and BMD, not with the bone isoenzyme; this could be due to the low precision of the electrophoretic method utilized for bALP determination. Unfortunately, these are the only variables in our possession for all the patients at the moment. The values of ALP were reduced in thalassemic patients vs normal controls. The discrepancy between osteocalcin and ALP could be explained as stated in the revised paper, page 7, lines 1 to 3 from the bottom: "In effect these parameters, though both related to bone mineralization, differ as alkaline phosphatase assesses osteoblastic activity, osteocalcin expresses bone mineralization. It is well known that mineralization is defective in thalassemic patients (references no 16 and 17 of the revised paper), while data on bone formation are contrasting."

3) Control values (and not the expected values by the manufacturer of the kits) are needed for all studied parameters.

- A matched control group was included and the values for the measured parameters were compared with those obtained in the thalassemic patients.

4) DEXA has to be substituted by DXA throughout the text.

- The term was substituted throughout the text. 
Conflict of interest

Click here to download Conflict of interest: conflitto interessi-1.jpg 
Dear Sir, we are grateful for the useful suggestions of the reviewers. We have revised our paper following their recommendations.

We trust the paper in the present form to be suitable for publication.

Reviewers' comments:

In the Discussion session they comment that "....the correlation between alkaline phosphatase and bone mineralization indicates a prevalent role of reduced deposition over increased reabsorption, as reported by other Authors, at variance with others. We could not confirm the same relationship with osteocalcin, another marker of osteoblastic activity.".

First of all the authors have not measured any of the markers of bone resorption. I believe that it is important for the paper and for the the discussion to include at least NTX or CTX measurements. I hope that they have available serum for such measurement. I suggest that CTX is more sensitive than NTX in the serum.

In the original paper, we did not include any marker of bone resorption because we had only partial data (different markers, incomplete series). It seems now evident that the importance of these indices to our work has been understated. In effect, we had available serum of most patients, so we decided to measure CTX following the useful suggestion of the reviewer; we recognize that this adjunct increases the scientific interest of our results.

As pointed out in the revised paper (page 8, lines 1 to 3) "no significant differences were found in CTX serum levels between thalassemic patients and controls, nor significant correlations with any of the studied parameters. This result may be due to the wide variability of the values in both cohorts; the problem should probably be obviated by a longitudinal study protocol."

Second, in order to state that the correlation of bALP with BMD indicates a prevalent role of reduced deposition, someone could expect a strong correlation. On the contrary the correlation was weak $(r<-0.25)$. Furthermore, we do not know if the bALP values were reduced (that will indicate reduced formation) or not.

The correlation was found between total ALP and BMD, not with the bone isoenzyme; this could be due to the low precision of the electrophoretic method utilized for bALP determination.

Unfortunately, these are the only variables in our possession for all the patients at the moment. The values of ALP were reduced in thalassemic patients vs normal controls. The discrepancy between osteocalcin and ALP could be explained as stated in the revised paper, page 7, lines 1 to 3 from the bottom: "In effect these parameters, though both related to bone mineralization, differ as alkaline phosphatase assesses osteoblastic activity, osteocalcin expresses bone mineralization. It is well known that mineralization is defective in thalassemic patients (references no 16 and 17 of the revised paper), while data on bone formation are contrasting."

Control values (and not the expected values by the manufacturer of the kits) are needed for all studied parameters.

A matched control group was included and the values for the measured parameters were compared with those obtained in the thalassemic patients.

DEXA has to be substituted by DXA throughout the text.

The term was substituted throughout the text. 


\section{Endocrine and bone disease in appropriately treated adult patients with beta-Thalassemia} major

Marina Baldini ${ }^{\mathrm{a}}$, Stella Forti ${ }^{\mathrm{b}}$, Alessia Marcon ${ }^{\mathrm{a}}$, Fabio Massimo Ulivieri ${ }^{\mathrm{d}}$, Alessandra Orsatti ${ }^{\mathrm{c}}$, Benedetta Tampieri $^{\mathrm{a}}$, Lorena Airaghi ${ }^{\mathrm{e}}$, Laura Zanaboni ${ }^{\mathrm{a}}$, Maria Domenica Cappellini ${ }^{\mathrm{a}}$

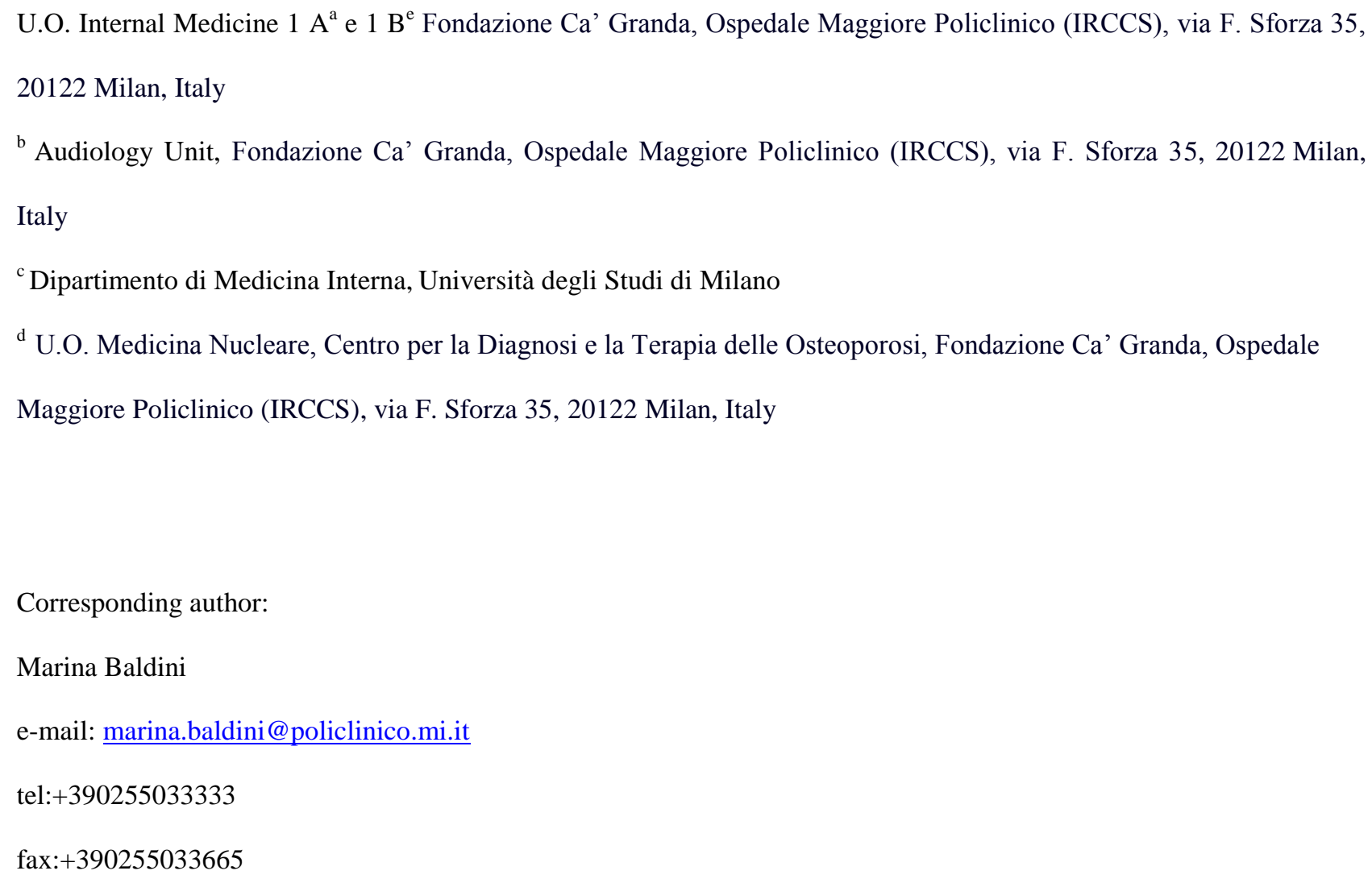




\begin{abstract}
With optimization of transfusional and chelation regimens, beta Thalassemia has changed from a pediatric disease with poor life expectancy into a chronic disease. Bone demineralization is an important cause of morbidity in older patients; the etiology is multifactorial and partially unknown. We examined cross-sectionally 111 adult patients with betaThalassemia Major (66 females and 45 males, $32.6 \pm 6$ years), regularly transfused, sufficiently chelated and replaced for endocrine defects. Bone demineralization was detected in $92.7 \%$ of patients, with different severity according to gender and site: osteopenia was the prominent finding at the femur, osteoporosis at lumbar spine $(\mathrm{p}<0.001)$, more evident in the males. The femoral site was more influenced by biochemical and clinical factors; despite adequate replacement, femoral T-score was lower in the hypogonadic than in the eugonadic group $(\mathrm{p}=0.047)$. A significant correlation was found between bone mass and body mass index (BMI), alkaline phosphatase (ALP) and pretransfusional $\mathrm{Hb}$ levels. The multivariate analysis indicated as significant regressors ALP, BMI and hypoparathyroidism (T-score: $\mathrm{p}=0.005,0.035$ and 0.002; Z-score: 002, 0.009, 0.003, respectively) at the femoral site, only ALP at the lumbar spine $(\mathrm{p}=0.008$ and 0.045 for $\mathrm{T}$ and $\mathrm{Z}$ scores, respectively). The statistical significance was reached more frequently by T-score, while Z-score seemed to be a less sensitive parameter.

Despite best care facilities, bone demineralization in thalassemic patients remains a challenge; further exploration of the relationships between bone loss and endocrine, biochemical and hematologic parameters is warranted to find effective measures to reduce fracture risk in this disease.
\end{abstract}

Key words: Thalassemia, osteoporosis, iron overload, endocrinopathy. 


\section{Introduction}

The life expectancy of patients affected by Thalassemia Major (TM) is significantly increased during the last decade due to regular transfusion regimen associated to adequate iron chelation. Today most of the patients enter adult life with a relevant improvement of quality of life[1]. Bone deformities, which could not be forecasted 20 years ago are quite rare today, nevertheless, low bone mass still remains a significant cause of morbidity in adult patients with TM. An increased prevalence of osteoporosis, and consequently of fractures due to bone fragility, continues to be reported [2,3] despite transfusions, effective chelation, calcium and vitamin D supplementation and replaced hormonal deficiencies. Several concomitant factors may contribute to the pathogenesis of osteoporosis in adult TM patients, making difficult a proper and preventing therapeutic approach.

We designed a cross-sectional evaluation of adult Thalassemia Major patients followed-up at single tertiary outpatient clinic in Milan, with optimal transfusional and therapeutical regimen, in order to assess the prevalence of bone demineralization and the relationships between bone status and physiological and pathological factors, including ferritin, pre-transfusional haemoglobin levels, bone metabolism markers and endocrine complications. 


\section{Patients}

After the approval of the Ethical Committee of our Institution and after patient's informed consent, 111 adult Caucasian patients affected by beta-Thalassemia Major (homozygous or double heterozygous for severe beta mutations) were enrolled in this study (66 females and 45 males, mean age $32.6 \pm 6$ years (33, range 19-50). All the patients received regular blood transfusions every 20-25 days, maintaining pre-transfusional haemoglobin around 9-9.5 g/L, according to the international guidelines [4]. Chelation therapy included deferoxamine (DFO) (39 pts), deferiprone (DFP) (7 pts), deferasirox (DFX)(46 pts) or combination DFO/DFP (19 pts). The chelation adequacy was estimated based on mean ferritin levels every 3 months and LIC by MRI when available. Exclusion criteria were medical conditions and pharmacological treatments interfering with the bone mineral metabolism.

The study design was cross-sectional; a periodic endocrinological evaluation was planned and during the same day bone densitometry, blood and urine biochemical tests and blood hormone evaluations were performed. Data collected included age, gender, BMI, history of auxologic and sexual development, splenectomy, fractures and liver disease, drugs used, transfusion regimen and starting age, mean of pre-transfusional haemoglobin and ferritin in the previous year, liver function, endocrine tests and bone metabolism indices, bone densitometry of femur and lumbar spine. A control group of healthy subjects ( 24 men and 35 women, mean age $33.7 \pm 6.8$ years) was recruited and compared with thalassemic patients for bone metabolism indices.

At the time of the biochemical and densitometric assessment, all hypogonadal patients were on hormonal replacement therapy, and all hypothyroid ones were on replacement with levothyroxine. Patients with hypoparathyroidism were treated with conventional vitamin D and calcium supplements, at a dose sufficient to maintain serum calcium within the normal range or at the lower normal limit.

\section{Methods}

Liver and renal function tests, calcium and phosphorus in serum (n. v. 2.1-2.5 and 0.8-1.4 mmol/L, respectively) and in 24-hour urine (n.v. 2.5-7.5 mmol/d and 12.9-42 mmol/d, respectively), intact parathyroid hormone (n.v. 1.5-6.6 pmol/L), osteocalcin (n.v. females 5.3-23.6, mcg/l, males 4.4-26.1), 25-hydroxyvitamin D (n.v. > 72 nmol/L), ferritin (n.v. 30-400 mcg/L) and gamma-glutamyiltranspeptidase (8-62 U/L) were determined by automated routine procedures. Blood cell count was performed by the Coulter Counter. Alkaline phosphatase was measured by IFCC liquid (n.v. 40$129 \mathrm{U} / \mathrm{L})$, while the bone isoenzyme was assessed by semiquantitative electrophoretic method with scanning densitometer (n.v. 20-75\%); serum CTX was determined by the Serum Cross Laps OneStep enzyme-linked immunosorbent assay (ELISA, IDS, Boldon, Tyne \& Wear, UK; n.v.: women, post-menopausal 0.142-1.351, premenopausal 0.112-0.73; males $0.115-0.748 \mathrm{ng} / \mathrm{mL}$ ) 
Iron overload was properly estimated by Liver Iron Content (LIC) measured by MRI [5], that showed a wide standard deviation due to the patients' compliance and the different efficacy of therapies.

Bone mineral density measurement was performed by means of dual x-ray photon absorptiometry (DXA) using Hologic bone densitometer (Hologic QDR-Discovery A, Version 12.7.3.1).

BMD values were expressed as T-score and Z-score. T-score was calculated as SDs from normal reference population database, while Z-score as SDs from age and sex matched population.

Data were classified according to WHO report (WHO Technical Report, ISCD Official Position Paper 2007); T-score > -1: normal. $-1>$ T-score $>-2.5$ : low bone density (osteopenia). T-score $<-2.5$ : osteoporosis.

\section{Statistical analysis}

Data are presented as means \pm SD. Intergroup comparison was performed with the Mann-Whitney test. Associations between parameters were determined by Pearson's and Spearman's rank correlation coefficients. To find independent factors affecting bone mineral density in thalassemic patients, a step-wise linear regression analysis was conducted. Two-sided exact tests were used and $p$ values of less than 0.05 were considered significant. All statistics were calculated using the Statistical Package for the Social Sciences 17.0 for Windows software package (SPSS Inc, Chicago, IL). 


\section{Results}

The demographic characteristics of the patients studied are presented in Table 1. All the patients were on iron chelating treatment: $35 \%$ with deferoxamine $(30+5 \mathrm{mg} / \mathrm{Kg}), 42 \%$ with deferasirox $(20+15 \mathrm{mg} / \mathrm{Kg})$, $6 \%$ with deferiprone $(75 \mathrm{mg} / \mathrm{Kg}$ ) and the remaining $17 \%$ with combined therapy (deferoxamine $30 \mathrm{mg} / \mathrm{Kg}+$ deferiprone $75 \mathrm{mg} / \mathrm{Kg})$ ).

101 patients $(91 \%)$ were anti-HCV positive, of whom 50.5\% were HCV RNA positive. $68 \%$ were splenectomised.

\section{Evaluation of bone mineral metabolism and endocrine function}

The biochemical indices of bone metabolism are reported in Table 2. All the parameters resulted within the normal range apart from $25 \mathrm{OH}$ vitamin $\mathrm{D}$ serum levels, that were suboptimal in 21 patients even if 13 of them were supplemented with $10.000 \mathrm{IU} /$ week. The levels of alkaline phosphatase and osteocalcin in the control group (68.1 \pm 15.5 $\mathrm{U} / \mathrm{L}$ and $10.0 \pm 4.9 \mathrm{mcg} / \mathrm{L}$ respectively) were significant lower (both $\mathrm{p}<0.001)$ than in thalassaemic patients $(85.4 \pm$ 28.7 U/L and 23.4 \pm 30.1 respectively). Conversely, PTH serum concentrations were significantly higher in the control group $(3.8 \pm 1.2, p=0.007$ vs $3.3 \pm 2.2)$, according to the diagnosis of hypoparathyroidism in 19 out of 111 thalassemic patients $(17.1 \%)$. All the hypoparathyroid patients were on treatment with calcium and calcitriol at doses normalizing serum calcium and phosphate concentrations; all of them were also hypogonadic, except one, resulting $16.2 \%$ the association of these endocrine diseases. No difference was found for serum levels between thalassemic and control group $(0.42 \pm 0.31$ vs $0.46 \pm 0.28)$.

Hypogonadism (in all cases hypogonadotrophic) was the most frequent endocrine complication in our cohort of patients, affecting 87 patients (78.4\%); all the hypogonadic patients except $4(95.4 \%)$ were on replacement treatment starting from the age of $18.8 \pm 3$ years. The hypogonadic patients who were not on replacement, being a minority, were excluded from the analysis of densitometric values.

An impairment of glucose metabolism was present in a high proportion of patients, with diabetes mellitus in 20 (18\%) and IGT in 24 of them (22\%). IGF-1 serum concentrations were evaluated in 78 patients (70\%), and resulted lower than normal in 45 of them $(41 \%)$.

\section{Bone mass measurements}

When considering the bone mass measurements in the overall population enrolled, the prevalence of demineralization (including both osteopenia and osteoporosis) was 92.7\% (103 out of 111 thalassemic patients). Indeed, mineralization was normal only in 18 patients $(16.2 \%)$ at the vertebral site and in 21 patients $(18.9 \%)$ at the femoral site.

As shown in Table 3, there were no differences in bone mineralization between the hypogonadic and the eugonadic patients at the vertebral site, while at the femoral site the T-score was lower in the hypogonadic group, despite adequate replacement ( $\mathrm{p}=0.047$ ). Further, only at the femoral site, bone mass was significantly different by gender, as females showed lower mean values of $\mathrm{T}$ and $\mathrm{Z}$-scores. There were statistically significant differences in $\mathrm{T}$-score at both sites 
also for the other dicotomic variables examined (hypoparathyroidism, treatment with calcium, treatment with bisphosphonates), while the statistical significance was not reached in all the analyses using Z-score as dependent variable.

Figure 1 shows the distribution of bone mineralization state (normal / osteopenia / osteoporosis) according to gender and site. Osteopenia was the prominent finding at the femoral site (63 femoral $v$ s 40 vertebral site, $\mathrm{p}<0.001)$, while at the vertebral site osteoporosis was more prevalent (53 pts vs 27 pts), the difference was statistically significant $(\mathrm{p}<0.001)$. The same distribution was confirmed when the patients were separately considered according to gender, being more evident in the male group $(\mathrm{p}<0.001)$. The patient distributions according to bone mineralization were significantly different by gender only at the femoral site.

Table 4 shows the results of the correlation analysis (correlation indices and statistical significance, $p<0.05)$ between bone mineralization (expressed by $\mathrm{T}$ and $\mathrm{Z}$ scores) and clinical/biochemical parametric variables. BMI was positively correlated with bone mass at femoral site, while advancing age was negatively correlated only with T-score. No correlation was found between vertebral T-score and the same variables. Among the biochemical indices of bone metabolism examined, alkaline phosphatase was the only one significantly correlated both with femoral and vertebral mineralization, being inversely associated with bone mass. Pre-transfusional Hb levels were significantly associated to vertebral Z-score.

The correlations between bone mineralization and the other clinical and biochemical characteristics of the patients (age at first transfusion, age at beginning of chelation, diabetes, previous splenectomy, LIC, serum concentrations of ferritin, parathyroid hormone, bone alkaline phosphatase, osteocalcin, $25 \mathrm{OH}$ vitamin D, IGF-1, CTX, and calcium both in serum and urine) were not significant thus were not reported.

The multivariate analysis indicated as possible regressors alkaline phosphatase, BMI and hypoparathyroidism $(\mathrm{p}=0.005$, 0.035 and 0.002 for $\mathrm{T}$-score; $0.002,0.009,0.003$ for Z-score, respectively) at the femoral site, while alkaline phosphatase was the only one found regressor for $\mathrm{T}$ and $\mathrm{Z}$ scores (respectively $\mathrm{p}=0.008$ and 0.045 ) at the lumbar spine. 


\section{Discussion}

Despite the longer life expectancy of thalassaemia major patients due to proper transfusional and chelation regimens [1], endocrine complications and bone disease still remain important causes of morbidity and disability [2,3,6-9] . In this study we examined adult patients with Thalassemia Major regularly transfused and sufficiently chelated; the patients were cared for $13 \pm 3$ years (mean \pm SD) at a tertiary single Italian centre where the Thalassemia International Guidelines [4] for monitoring and treating Thalassemia and its complications are issued and implemented..

Despite the availability of the best care facilities, bone demineralization was detected in a high proportion of patients, involving both sites examined though with different severity: osteopenia prevailed at femoral site, while osteoporosis was more prominent at lumbar spine (prevalently constituted by trabecular bone). Overall, the femoral site seemed to be more likely than the vertebral site influenced by biochemical and clinical factors. The only exception was pretransfusional $\mathrm{Hb}$, showing a positive correlation with vertebral mineralization; this could be explained by the high degree of ineffective erythropoiesis in the spine that can occurs in thalassemic syndromes. This finding is consistent with the interesting hypothesis that the chronic request for blood cell production can play a role in the etiology of osteoporosis, through overstimulation of the hematopoietic system increasing the number of osteoclasts and osteoblasts resulting in accelerated bone turnover [10].

In our analyses the statistical significance was reached more frequently by T-score, while Z-score seemed to be a less sensitive parameter. There is a statistical explanation to this behaviour: the relatively small number of cases can render the statistical power insufficient to detect smaller changes in Z-score, a parameter with high baseline variability. However, considering the very low prevalence of the disease, this is a sufficiently large cohort, and quite homogeneous as regards therapeutical approach and clinical management.

The statistically significant negative association between T-score and treatment with calcium and with bisphosphonates was expected, and compatible with the prescription of such drugs to the patients with more severe demineralization; the therapeutic effect of these medications could not be examined in our study, as the protocol did not include a longitudinal evaluation. Other parameters correlated to demineralization were gender (females are more involved), age and BMI, similarly to healthy individuals.

Even if we cannot address the issue of the prominent cause of bone disease in Thalassemia [11], the correlation between alkaline phosphatase and bone mineralization indicates a prevalent role of reduced deposition over increased reabsorption, as reported by other Authors [12], at variance with others [13, 14] . We could not confirm the same relationship with osteocalcin, another marker of osteoblastic activity $[12,15]$. In effect these parameters, though both related to bone mineralization, differ as alkaline phosphatase assesses osteoblastic activity, osteocalcin expresses bone 
mineralization. It is well known that mineralization is defective in thalassemic patients [16, 17], while data on bone formation are contrasting.

As regards bone resorption indices, no significant differences were found in CTX serum levels between thalassemic patients and controls nor significant correlations with any of the studied parameters. These results may be due to the wide variability of the values in both cohorts; the problem would probably be obviated by a longitudinal study protocol. As far as the endocrine complications relevant to bone status are concerned, according to previous reports [18] in our study the hypogonadic patients of both genders were more affected from demineralization than the eugonadic, and, interestingly, the standard hormone replacement therapy (HRT) does not normalize bone condition. A possible explanation may be the concurrence of factors interfering with mineralization, such as vitamin D deficiency, impairing the therapeutic effects of sex steroid hormones and also of bisphosphonates. A recent report [19] stated that an optimal vitamin D repletion is an essential pre-requisite for maximal efficacy of the anti-resorptive drugs in post-menopausal osteoporosis; a similar situation could be hypothesized in thalassemic population. In effect, low serum vitamin D levels have been reported in thalassemic populations; this was ascribed to impaired liver hydroxylation and intestinal absorption, with a causative role of iron overload suggested by a negative correlation between serum 25-OH-vitamin D and ferritin [20]. This correlation was not confirmed in our study $(\mathrm{p}=0.546)$. Unexpectedly, in our series the serum vitamin D was often low despite ongoing treatment with metabolites, compatible with a defect in absorption or metabolism [20] but also with low compliance to the therapy; the latter is quite common using associations with calcium salts, badly tolerated. Anyway, the contribution of vitamin D to bone status is probably more complex than presently recognized, and neither vitamin D concentrations nor ongoing treatment did correlate with mineralization status in our and earlier reports [21]. Conversely, impairment of parathyroid function was inversely related to mineralization status, indicating a protective effect on bone of this endocrine complication [21, 22]. In our study the relationship was more evident at the femoral level than at lumbar spine; this was unexpected, as chronic PTHdeprivation should influence more strongly the sites where trabecular content is maximum. Probably, in this peculiar population the superimposed pathologic mechanisms concurring to bone disease can produce net effects difficult to predict in the long-term.

Finally, an important consideration emerges from our statistical analysis: the model utilized could account for the $29.4 \%$ of T-score variance at the femur and the $32.03 \%$ at the spine. This indicates that the variables significant in the univariate analysis could not explain satisfactorily the mineralization defect. Again, this may be ascribed to insufficient statistical power, linked to the restricted number of cases in a monocentric study, or alternatively to the influence of pathogenetically involved factors beyond those included in our analysis. The indices studied reflect bone marrow 
expansion, iron overload, endocrine function and bone metabolism, but further physiological, pathological, genetic and iatrogenic factors can work in this multifactorial disease.

Furthermore, early preventive intervention and management are needed to influence bone health in adulthood, as demonstrated by normal Z-scores found in children and young adolescents with Thalassemia Major by Christoforidis et al [23]. Our patients are probably comparable for the quality of clinical management; however the different results emerged from our study suggest that transfusion and chelation treatment can prevent bone demineralization only when applied early from childhood.

A deeper learning of the molecular mechanisms causing demineralization, as well as the optimal prevention and treatment of Thalassemia from infancy, could perhaps allow to avoid complications and further ameliorate the quality of life in these patients. 
Fig 1 Bone mineralization state (normal / osteopenia / osteoporosis) of the thalassemic patients as distributed on the basis of site and gender, according to the WHO osteoporosis classification. Osteopenia was the prominent finding at the femur, while at lumbar spine osteoporosis was more prevalent $(\mathrm{p}<0.001)$. The same distribution was confirmed when the patients were separately considered according to gender, being more evident in the male group

Table 1 Clinical characteristics of the patients by gender at the time when mineralization was studied

Table 2 Biochemical and hormonal indices related to bone metabolism in 111 patients affected by Thalassemia Major

Table 3 Statistic results (mean \pm standard deviation and $p$ value) of comparisons between clinical/biochemical dicotomic variables and $\mathrm{T}$ or $\mathrm{Z}$ - scores at femoral and vertebral sites

Table 4 Correlation analysis (Pearson's correlation indices and two-tailed $p$ values) between bone mineralization (expressed by $\mathrm{T}$ and Z-scores) and clinical/biochemical parametric variables 


\section{References}

1. Borgna-Pignatti C, Cappellini MD, De Stefano P, Del Vecchio GC, Forni GL, Gamberini MR, Ghilardi R, Origa R, Piga A, Romeo MA, Zhao H, Cnaan A (2005) Survival and complications in thalassemia. Ann N Y Acad Sci 1054:4047

2. Vogiatzi MG, Macklin EA, Fung EB, Vichinsky E, Olivieri N, Kwiatkowski J, Cohen A, Neufeld E, Giardina PJ (2006) Prevalence of fractures among the thalassemia syndromes in north america. Bone 38(4):571-575

3. Vogiatzi MG, Macklin EA, Fung EB, Cheung AM, Vichinsky E, Olivieri N, Kirby M, Kwiatkowski JL, Cunningham M, Holm IA, Lane J, Schneider R, Fleisher M, Grady RW, Peterson CC, Giardina PJ, Thalassemia Clinical Research Network (2009) Bone disease in thalassemia: A frequent and still unresolved problem. J Bone Miner Res 24(3):543-557

4. Cappellini M.D, Cohen A, Eleftheriou A, Piga A, Porter J, Taher A (2008) Guidelines for the clinical management of thalassemia; $2^{\text {nd }}$ revised Edition. Thalassemia International Federation. http://thalassemia.org.cy

5.Wood JC, Enriquez C, Ghugre N, Tyzka JM, Carson S, Nelson MD, Coates TD (2005) MRI R2 and R2* mapping accurately estimates hepatic iron concentration in transfusion-dependent thalassemia and sickle cell disease patients. Blood 106 (4):1460-65

6. Dubey AP, Parakh A, Dublish S (2008) Current trends in the management of beta thalassemia. Indian J Pediatr 75(7):739-743

7. Perrotta S, Cappellini MD, Bertoldo F, Servedio V, Iolascon G, D'Agruma L, Gasparini P, Siciliani MC, Iolascon A (2000) Osteoporosis in beta-thalassaemia major patients: Analysis of the genetic background. Br J Haematol 111(2):461-466

8. Origa R, Fiumana E, Gamberini MR, Armari S, Mottes M, Sangalli A, Paglietti E, Galanello R, Borgna-Pignatti C (2005) Osteoporosis in beta-thalassemia: Clinical and genetic aspects. Ann N Y Acad Sci 1054:451-456

9. Skordis N (2009) The labyrinth of bone disease in thalassaemia: The search for ariadne's thread continues. Eur J Haematol 82(1):13-14 
10. Gurevitch O, Slavin S (2006) The hematological etiology of osteoporosis. Med Hypotheses 67(4):729-735

11. Voskaridou E, Terpos E (2008) Pathogenesis and management of osteoporosis in thalassemia. Pediatr Endocrinol Rev 6 Suppl 1:86-93

12. Salama OS, Al-Tonbary YA, Shahin RA, Eldeen OA (2006) Unbalanced bone turnover in children with betathalassemia. Hematology 11(3):197-202

13. Dresner Pollack R, Rachmilewitz E, Blumenfeld A, Idelson M, Goldfarb AW (2000) Bone mineral metabolism in adults with beta-thalassaemia major and intermedia. Br J Haematol 111(3):902-907

14. Voskaridou E, Kyrtsonis MC, Terpos E, Skordili M, Theodoropoulos I, Bergele A, Diamanti E, Kalovidouris A, Loutradi A, Loukopoulos D (2001) Bone resorption is increased in young adults with thalassaemia major. Br J Haematol 112(1):36-41

15. Morabito N, Gaudio A, Lasco A, Atteritano M, Pizzoleo MA, Cincotta M, La Rosa M, Guarino R, Meo A, Frisina N (2004) Osteoprotegerin and RANKL in the pathogenesis of thalassemia-induced osteoporosis: New pieces of the puzzle. J Bone Miner Res 19(5):722-727

16. Hiratsuka H, Katsuta O, Toyota N, Tsuchitani M, Akiba T, Marumo F, Umemura T (1997) Iron deposition at mineralization fronts and osteoid formation following chronic cadmium exposure in ovariectomized rats. Toxicol Appl Pharmacol 143:348-356

17. Mahachoklertwattana P, Sirikulchayanonta V, Chuansumrit A, Karnsombat P, Choubtum L, Sriphrapradang A, Domrongkitchaiporn S, Sirisriro R, Rajatanavin R (2003) Bone histomorphometry in children and adolescents with beta-thalassemia disease: iron-associated focal osteomalacia. J Clin Endocrinol Metab 88 ( 8):3966-3972

18. Carmina E, Di Fede G, Napoli N, Renda G, Vitale G, Lo Pinto C, Bruno D, Malizia R, Rini GB (2004) Hypogonadism and hormone replacement therapy on bone mass of adult women with thalassemia major. Calcif Tissue Int 74(1):68-71

19. Adami S, Giannini S, Bianchi G, Sinigaglia L, Di Munno O, Fiore CE, Minisola S, Rossini M (2009) Vitamin D status and response to treatment in post-menopausal osteoporosis. Osteoporos Int 20(2):239-244 
20. Napoli N, Carmina E, Bucchieri S, Sferrazza C, Rini GB, Di Fede G (2006) Low serum levels of 25-hydroxy

1 vitamin D in adults affected by thalassemia major or intermedia. Bone 38(6):888-892

21. Angelopoulos NG, Goula A, Rombopoulos G, Kaltzidou V, Katounda E, Kaltsas D, Tolis G (2006)

Hypoparathyroidism in transfusion-dependent patients with beta-thalassemia. J Bone Miner Metab 24(2):138-145

22. Laway BA, Goswami R, Singh N, Gupta N, Seith A (2006) Pattern of bone mineral density in patients with sporadic idiopathic hypoparathyroidism. Clin Endocrinol (Oxf) 64(4):405-409

23. Christoforidis A, Kazantzidou E, Tsatra I, Tsantali H, Koliakos G, Hatzipantelis E, Katzos G, Athanassiou-Metaxa M (2007) Normal lumbar bone mineral density in optimally treated children and young adolescents with betathalassaemia major. Hormones (Athens) 6(4):334-340 


\section{General}

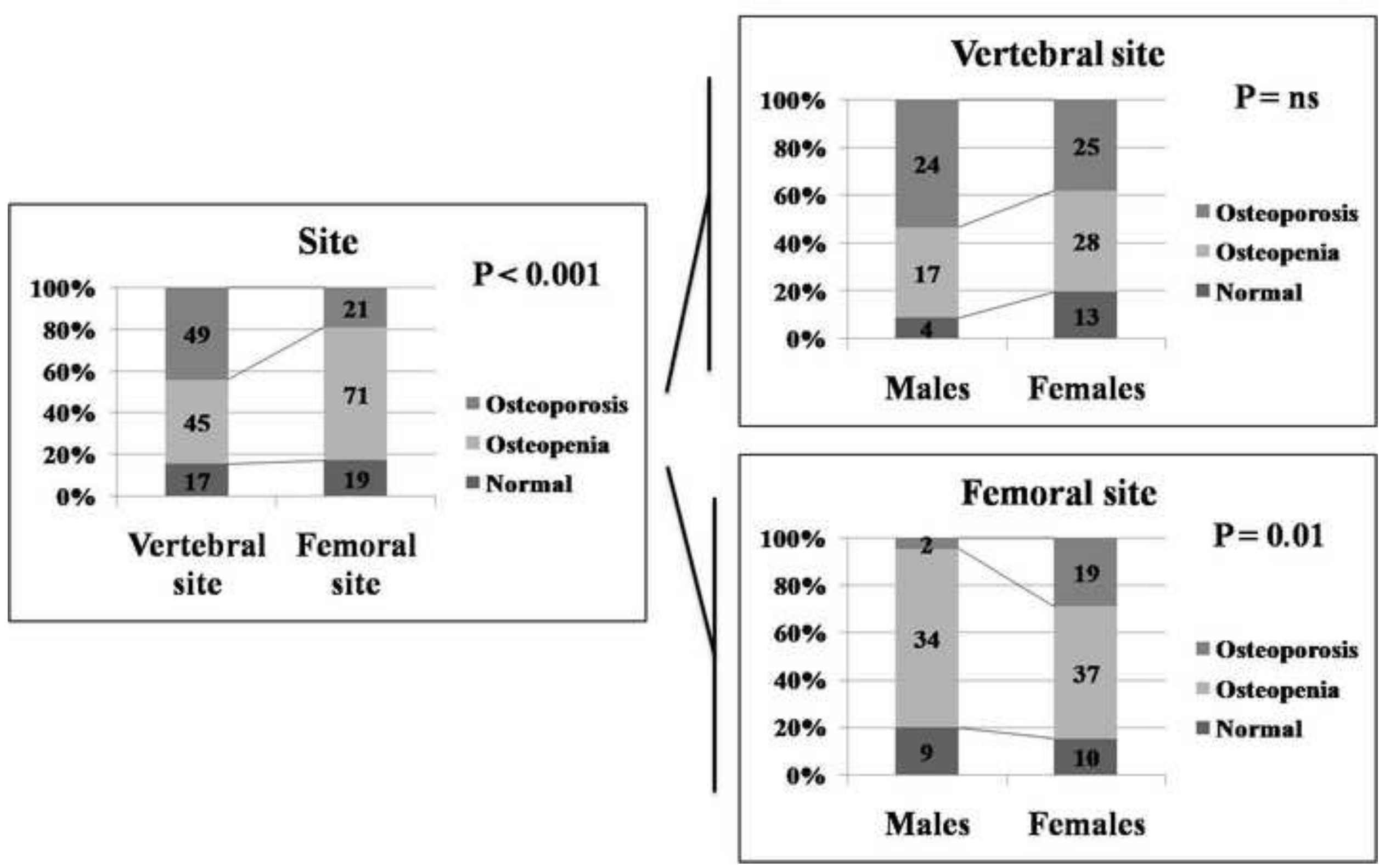

\section{By gender}




\begin{tabular}{|l|c|c|c|c|}
\hline & \multicolumn{2}{|c|}{ males } & \multicolumn{2}{c|}{ females } \\
\hline & Mean \pm SD & range & Mean \pm SD & range \\
\hline Age (years) & $32.5 \pm 4.9$ & $22-44$ & $33.9 \pm 6.0$ & $19-46$ \\
\hline Age at first transfusion (years) & $14.3 \pm 11.8$ & $4-48$ & $17.3 \pm 16.0$ & $3-60$ \\
\hline Age at beginning chelation (years) & $5.2 \pm 3.7$ & $1-15$ & $5.8 \pm 3.5$ & $0-15$ \\
\hline Body Mass Index (kg/m $\left.{ }^{2}\right)$ & $22.9 \pm 3.3$ & $16-29$ & $22.3 \pm 3.0$ & $18-31$ \\
\hline Pre-transfusion hemoglobin (g/dl) & $9.4 \pm 0.6$ & $8.4-10.4$ & $9.3 \pm 0.6$ & $8-10.4$ \\
\hline Serum ferritin (mcg/L) & $1267 \pm 1168$ & $431-6563$ & $1452 \pm 912$ & $354-4509$ \\
\hline Liver Iron Content (mg Fe/g liver d.w.) & $5.1 \pm 3.9$ & $0.6-19.3$ & $5.8 \pm 3.7$ & $0.6-15.9$ \\
\hline
\end{tabular}




\begin{tabular}{|l|c|c|c|}
\hline & mean \pm SD & range & median \\
\hline Serum calcium (mmol/L) & $2.2 \pm 0.1$ & $1.6-2.6$ & 9 \\
\hline Serum phosphate (mmol/L) & $1.3 \pm 0.1$ & $0.7-2.2$ & 1.2 \\
\hline Urinary calcium (mmol/d) & $6.3 \pm 3.7$ & $0.4-17$ & 5.8 \\
\hline Urinary phosphate (mmol/d) & $22.6 \pm 9.7$ & $3.2-83.9$ & 22.6 \\
\hline Alkaline phosphatase (U/L) & $85.4 \pm 28.7$ & $33-204$ & 85 \\
\hline Bone alkaline phosphatase (\%) & $62 \pm 17$ & $7-95$ & 65 \\
\hline GGT (U/L) & $21.2 \pm 12.9$ & $7-72$ & 35 \\
\hline Osteocalcin (mcg/L) & $23.4 \pm 30.1$ & $0.3-209$ & 14.2 \\
\hline Parathyroid hormone (pmol/L) & $3.3 \pm 2.2$ & $0.2-14.9$ & 2.9 \\
\hline 25OH Vitamin D (nmol/L) & $46.8 \pm 29.3$ & $9.6-132$ & 38.6 \\
\hline CTX (ng/ml) & $0.4 \pm 0.3$ & $0.1-1.4$ & 0.3 \\
\hline
\end{tabular}




\begin{tabular}{|c|c|c|c|c|c|c|c|c|c|}
\hline & & \multicolumn{4}{|c|}{ T-score } & \multicolumn{4}{|c|}{ Z-score } \\
\hline & & Femoral & & Vertebr: & & Femoral & & Vertebra & \\
\hline & & Mean $\pm S D$ & $p$ & Mean $\pm S D$ & $p$ & Mean $\pm S D$ & $p$ & Mean $\pm S D$ & $p$ \\
\hline Gender & $\begin{array}{l}\text { males } \\
\text { females }\end{array}$ & $\begin{array}{l}-1.58 \pm 0.746 \\
-1.90 \pm 1.018\end{array}$ & 0.046 & $\begin{array}{l}-2.44 \pm 1.119 \\
-2.12 \pm 1.267\end{array}$ & $n s$ & $\begin{array}{l}-1.50 \pm 0.830 \\
-1.87 \pm 0.967\end{array}$ & 0.027 & $\begin{array}{l}-2.28 \pm 1.196 \\
-2.04 \pm 1.219\end{array}$ & ns \\
\hline Hypogonadism & $\begin{array}{l}\text { no } \\
\text { yes }\end{array}$ & $\begin{array}{l}-1.46 \pm 0.772 \\
-1.85 \pm 0.952\end{array}$ & 0.047 & $\begin{array}{l}-2.20 \pm 1.095 \\
-2.26 \pm 1.250\end{array}$ & $n s$ & $\begin{array}{l}-1.65 \pm 0.744 \\
-1.73 \pm 0.975\end{array}$ & ns & $\begin{array}{l}-2.12 \pm 1.098 \\
-2.15 \pm 1.247\end{array}$ & ns \\
\hline Hypoparathyroidism & $\begin{array}{l}\text { no } \\
\text { yes }\end{array}$ & $\begin{array}{l}-1.87 \pm 0.855 \\
-1.31 \pm 1.130\end{array}$ & 0.021 & $\begin{array}{l}-2.36 \pm 1.116 \\
-1.71 \pm 1.527\end{array}$ & 0.039 & $\begin{array}{l}-1.82 \pm 0.850 \\
-1.20 \pm 1.118\end{array}$ & 0.017 & $\begin{array}{l}-2.21 \pm 1.126 \\
-1.79 \pm 1.547\end{array}$ & ns \\
\hline Calcium treatment & $\begin{array}{l}\text { no } \\
\text { yes }\end{array}$ & $\begin{array}{l}-1.64 \pm 0.819 \\
-1.93 \pm 1.030\end{array}$ & 0.035 & $\begin{array}{l}-2.05 \pm 1.160 \\
-2.49 \pm 1.247\end{array}$ & 0.036 & $\begin{array}{l}-1.68 \pm 0.795 \\
-1.75 \pm 1.081\end{array}$ & ns & $\begin{array}{l}-2.01 \pm 1.192 \\
-2.31 \pm 1.224\end{array}$ & $n s$ \\
\hline Bisphosphonate treatment & $\begin{array}{l}\text { no } \\
\text { yes }\end{array}$ & $\begin{array}{l}-1.67 \pm 0.905 \\
-2.35 \pm 0.674\end{array}$ & 0.003 & $\begin{array}{l}-2.09 \pm 1.190 \\
-3.23 \pm 0.634\end{array}$ & $<0.001$ & $\begin{array}{l}-1.66 \pm 0.928 \\
-2.14 \pm 0.823\end{array}$ & 0.041 & $\begin{array}{l}-1.99 \pm 1.184 \\
-3.16 \pm 0.614\end{array}$ & 0.001 \\
\hline
\end{tabular}




\begin{tabular}{|c|c|c|c|c|c|c|c|c|}
\hline & \multicolumn{4}{|c|}{ T - score } & \multicolumn{4}{|c|}{ Z-score } \\
\hline & Femoral & & Vertebral & & Femoral & & Vertebral & \\
\hline & Correlation index & $p$ & Correlation index & $p$ & Correlation index & $p$ & Correlation index & $p$ \\
\hline Age & -0.218 & 0.022 & -0.032 & $n s$ & -0.021 & $n s$ & 0.063 & $n s$ \\
\hline BMI & 0.259 & 0.006 & 0.064 & $n s$ & 0.276 & 0.006 & 0.049 & $n s$ \\
\hline Pre-transfusional hemoglobin & -0.088 & $n s$ & 0.179 & $n s$ & -0.058 & $n s$ & 0.198 & 0.042 \\
\hline Alkaline Phosphatase & -0.223 & 0.019 & -0.233 & 0.014 & -0.024 & 0.024 & 0.137 & $n s$ \\
\hline CTX & 0.032 & $n s$ & -0.028 & $n s$ & -0.024 & $n s$ & -0.047 & $n s$ \\
\hline
\end{tabular}




\section{Endocrine and bone disease in appropriately treated adult patients with beta-thalassemia major}

Marina Baldini ${ }^{\mathrm{a}}$, Stella Forti ${ }^{\mathrm{b}}$, Alessia Marcon ${ }^{\mathrm{a}}$, Fabio Massimo Ulivieri $^{\mathrm{d}}$, Alessandra Orsatti ${ }^{\mathrm{c}}$, Benedetta Tampieri $^{\mathrm{a}}$, Lorena Airaghi ${ }^{\mathrm{e}}$, Laura Zanaboni ${ }^{\mathrm{a}}$, Maria Domenica Cappellini ${ }^{\mathrm{a}}$

U.O. Medicina Interna $1 \mathrm{~A}^{\mathrm{a}}$ e $1 \mathrm{~B}^{\mathrm{e}}$ Fondazione Ca' Granda, Ospedale Maggiore Policlinico (IRCCS), via F. Sforza 35, 20122 Milan, Italy

${ }^{\mathrm{b}}$ Audiologia, Fondazione Ca' Granda, Ospedale Maggiore Policlinico (IRCCS), via F. Sforza 35, 20122 Milan, Italy

${ }^{c}$ Dipartimento di Medicina Interna, Università degli Studi di Milano

${ }^{\text {d }}$ U.O. Medicina Nucleare, Centro per la Diagnosi e la Terapia delle Osteoporosi, Fondazione Ca' Granda, Ospedale Maggiore Policlinico (IRCCS), via F. Sforza 35, 20122 Milan, Italy

Corresponding author:

Marina Baldini

e-mail: marina.baldini@policlinico.mi.it

tel: +390255033333

fax:+390255033665 


\begin{abstract}
With optimization of transfusional and chelation regimens, beta thalassemia has changed from a pediatric disease with poor life expectancy into a chronic disease. Bone demineralization is an important cause of morbidity in older patients; the etiology is multifactorial and partially unknown. We examined cross-sectionally 111 adult patients with betaThalassemia Major (66 females and 45 males, $32.6 \pm 6$ years), regularly transfused, sufficiently chelated and replaced for endocrine defects. Bone demineralization was detected in $92.7 \%$ of patients, with different severity according to gender and site: osteopenia was the prominent finding at the femur, osteoporosis at lumbar spine $(\mathrm{p}<0.001)$, more evident in the males. The femoral site was more influenced by biochemical and clinical factors; despite adequate replacement, femoral T-score was lower in the hypogonadic than in the eugonadic group $(\mathrm{p}=0.047)$. A significant correlation was found between bone mass and body mass index (BMI), alkaline phosphatase (ALP) and pretransfusional $\mathrm{Hb}$ levels. The multivariate analysis indicated as significant regressors ALP, BMI and hypoparathyroidism (T-score: $\mathrm{p}=0.005,0.035$ and 0.002; Z-score: 002, 0.009, 0.003, respectively) at the femoral site, only ALP at the lumbar spine $(\mathrm{p}=0.008$ and 0.045 for $\mathrm{T}$ and $\mathrm{Z}$ scores, respectively). The statistical significance was reached more frequently by T-score, while Z-score seemed to be a less sensitive parameter.

Despite best care facilities, bone demineralization in thalassemic patients remains a challenge; further exploration of the relationships between bone loss and endocrine, biochemical and hematologic parameters is warranted to find effective measures to reduce fracture risk in this disease.
\end{abstract}

Key words: Thalassemia, osteoporosis, iron overload, endocrinopathy. 


\section{Introduction}

The life expectancy of patients affected by thalassemia major (TM) is significantly increased during the last decade due to regular transfusion regimen associated to adequate iron chelation . Today most of the patients enter adult life with a relevant improvement of quality of life[1]. Bone deformities, which could not be forecasted 20 years ago are quite rare today, nevertheless, low bone mass still remains a significant cause of morbidity in adult patients with TM. An increased prevalence of osteoporosis, and consequently of fractures due to bone fragility, continues to be reported [2,3] despite transfusions, effective chelation, calcium and vitamin D supplementation and replaced hormonal deficiencies. Several concomitant factors may contribute to the pathogenesis of osteoporosis in adult TM patients, making difficult a proper and preventing therapeutic approach.

We designed a cross-sectional evaluation of adult thalassemia major patients followed-up at single tertiary outpatient clinic in Milan, with optimal transfusional and therapeutical regimen, in order to assess the prevalence of bone demineralization and the relationships between bone status and physiological and pathological factors, including ferritin, pre-transfusional haemoglobin levels, bone metabolism markers and endocrine complications. 


\section{Patients}

After the approval of the Ethical Committee of our Institution and after patient's informed consent, 111 adult Caucasian patients affected by beta-Thalassemia Major (homozygous or double heterozygous for severe beta mutations) were enrolled in this study (66 females and 45 males, mean age $32.6 \pm 6$ years (33, range 19-50). All the patients received regular blood transfusions every 20-25 days, maintaining pre-transfusional haemoglobin around 9-9.5 g/L, according to the international guidelines [4]. Chelation therapy included deferoxamine (DFO) (39 pts), deferiprone (DFP) (7 pts), deferasirox (DFX)(46 pts) or combination DFO/DFP (19 pts). The chelation adequacy was estimated based on mean levels every 3 months and LIC by MRI when available. Exclusion criteria were medical conditions and pharmacological treatments interfering with the bone mineral metabolism.

The study design was cross-sectional; a periodic endocrinological evaluation was planned and during the same day bone densitometry, blood and urine biochemical tests and blood hormone evaluations were performed. Data collected included age, gender, BMI, history of auxologic and sexual development; splenectomy, fractures and liver disease, drugs used, transfusion regimen and starting age, mean of pre-transfusional haemoglobin and ferritin in the previous year, liver function and endocrine tests, bone densitometry of femur and lumbar spine.

At the time of the biochemical and densitometric assessment, all hypogonadal patients were on hormonal replacement therapy, and all hypothyroid ones were on replacement with levothyroxine. Patients with hypoparathyroidism were treated with conventional vitamin D and calcium supplements, at a dose sufficient to maintain serum calcium within the normal range or at the lower normal limit.

\section{Methods}

Liver and renal function tests, calcium and phosphorus in serum (n. v. 2.1-2.5 and 0.8-1.4 mmol/L, respectively) and in 24-hour urine (n.v. 2.5-7.5 mmol/d and 12.9-42 mmol/d, respectively), intact parathyroid hormone (n.v. 1.5-6.6 pmol/L), osteocalcin (n.v.14-42 mcg/L), 25-hydroxyvitamin D (n.v. > 72 nmol/L), ferritin (n.v. 30-400 mcg/L) were determined by automated routine procedures. Blood cell count was performed by Coulter Counter; alkaline phosphatase was measured by IFCC liquid (n.v. 40-129 U/L).

Iron overload was properly estimated by Liver Iron Content (LIC) measured by MRI [5], that showed a wide standard deviation due to the patients' compliance and the different efficacy of therapies..

Bone mineral density measurement was performed by means of dual x-ray photon absorptiometry (DEXA) using Hologic bone densitometer (Hologic QDR-Discovery A, Version 12.7.3.1).

BMD values were expressed as T-score and Z-score. T-score was calculated as SDs from normal reference population database, while Z-score as SDs from age and sex matched population. 
Data were classified according to WHO report (WHO Technical Report, ISCD Official Position Paper 2007); T-score >

\begin{abstract}
Statistical analysis
Data are presented as means \pm SD. Intergroup comparison was performed with the Mann-Whitney test. Associations between parameters were determined by Pearson's and Sperman's rank correlation coefficients. To find independent factors affecting bone mineral density in thalassemic patients, a step-wise linear regression analysis was conducted. Two-sided exact tests were used and $p$ values of less than 0.5 were considered significant. All statistics were calculated using the Statistical Package for the Social Sciences 17.0 for Windows software package (SPSS Inc, Chicago, IL).
\end{abstract}




\section{Results}

The demographic characteristics of the patients studied are presented in Table 1

All the patients were on iron chelating treatment: $35 \%$ with deferoxamine $(30+5 \mathrm{mg} / \mathrm{Kg}), 42 \%$ with deferasirox $(20+15 \mathrm{mg} / \mathrm{Kg}), 6 \%$ with deferiprone $(75 \mathrm{mg} / \mathrm{Kg})$ and the remaining $17 \%$ with combined therapy (deferoxamine 30mg/Kg + Deferiprone $75 \mathrm{mg} / \mathrm{Kg})$ ).

101 patients (91\%) were anti-HCV positive, of whom 50.5\% were HCV RNA positive. $68 \%$ were splenectomised.

\section{Evaluation of bone mineral metabolism and endocrine function}

The biochemical indices of bone metabolism are reported in Table 2. All the parameters resulted within the normal range apart from $250 \mathrm{OH}$ vitamin D serum levels, that were suboptimal in 21 patients even if 13 of them were supplemented with $10.000 \mathrm{IU} /$ week.

Nineteen out of 111 patients $(17.1 \%)$ were affected by hypoparathyroidism and treated with calcium and calcitriol at doses normalizing serum calcium and phosphate concentrations. All the hypoparathyroid patients were also hypogonadic, except one, resulting $16.2 \%$ the association of these endocrine diseases.

Hypogonadism (in all cases hypogonadotrophic) was the most frequent endocrine complication in our cohort of patients, affecting 87 patients (78.4\%); all the hypogonadic patients except $4(95.4 \%)$ were on replacement treatment starting from the age of $18.8 \pm 3$ years. The hypogonadic patients who were not on replacement, being a minority, were excluded from the analysis of densitometric values.

An impairment of glucose metabolism was present in a high proportion of patients, with diabetes mellitus in 20 (18\%) and IGT in 24 of them (22\%). IGF-1 serum concentrations were evaluated in 78 patients (70\%), and resulted lower than normal in 45 of them $(41 \%)$.

\section{Bone mass measurements}

When considering the bone mass measurements in the overall population enrolled, the prevalence of demineralization (including both osteopenia and osteoporosis) was 92.7\% (103 out of 111 Thalassemic patients). Indeed, mineralization was normal only in 18 patients $(16.2 \%)$ at the vertebral site and in 21 patients $(18.9 \%)$ at the femoral site.

As shown in Table 3, there were no differences in bone mineralization between the hypogonadic and the eugonadic patients at the vertebral site, while at the femoral site the T-score was lower in the hypogonadic group, despite adequate replacement ( $\mathrm{p}=0.047$ ). Further, only at the femoral site, bone mass was significantly different by gender, as females showed lower mean values of $\mathrm{T}$ and $\mathrm{Z}$-scores. There were statistically significant differences in $\mathrm{T}$-score at both sites also for the other dicotomic variables examined (hypoparathyroidism, treatment with calcium, treatment with bisphosphonates), while the statistical significance was not reached in all the analyses using Z-score as dependent variable. 
Figure 1 shows the distribution of bone mineralization state (normal / osteopenia / osteoporosis) according to gender and site. Osteopenia was the prominent finding at the femoral site (63 femoral $v$ s 40 vertebral site, $\mathrm{p}<0.001)$, while at the vertebral site osteoporosis was more prevalent (53 pts vs $27 \mathrm{pts}$ ), the difference was statistically significant $(\mathrm{p}<0.001)$. The same distribution was confirmed when the patients were separately considered according to gender, being more evident in the male group $(\mathrm{p}<0.001)$. The patient distributions according to bone mineralization were significantly different by gender only at the femoral site.

Table 4 shows the results of the correlation analysis (correlation indices and statistical significance, p<0.05) between bone mineralization (expressed by $\mathrm{T}$ and $\mathrm{Z}$ scores) and clinical/biochemical parametric variables. BMI was positively correlated with bone mass at femoral site, while advancing age was negatively correlated only with T-score. No correlation was found between vertebral T-score and the same variables. Among the biochemical indices of bone metabolism examined, alkaline phosphatase was the only one significantly correlated both with femoral and vertebral mineralization, being inversely associated with bone mass. Pre-transfusional Hb levels were significantly associated to vertebral Z-score.

The correlations between bone mineralization and the other clinical and biochemical characteristic of the patients (age at first transfusion, age at beginning of chelation, diabetes, previous splenectomy, LIC, serum concentrations of ferritin, parathyroid hormone, bone alkaline phosphatase, osteocalcin, $25 \mathrm{OH}$ vitamin D, IGF-1, and calcium both in serum and urine) were not significant thus were not reported.

The multivariate analysis indicated as possible regressors alkaline phosphatase, BMI and hypoparathyroidism $(\mathrm{p}=0.005$, 0.035 and 0.002 for T-score; 0.002, $0.009,0.003$ for Z-score, respectively) at the femoral site, while alkaline phosphatase was the only one found regressor for $\mathrm{T}$ and $\mathrm{Z}$ scores (respectively $\mathrm{p}=0.008$ and 0.045 ) at the lumbar spine. 


\section{Discussion}

Despite the longer life expectancy of thalassaemia major patients due to proper transfusional and chelation regimens ([1], endocrine complications and bone disease still remain important causes of morbidity and disability[2,3,6-9] . In this study we examined adult patients with thalassemia major regularly transfused and sufficiently chelated; the patients were cared for $13 \pm 3$ years (mean $\pm \mathrm{SD}$ ) at a tertiary single Italian centre where the Thalassemia International Guidelines [4] for monitoring and treating Thalassemia and its complications are issued and implemented..

Despite the availability of the best care facilities, bone demineralization was detected in a high proportion of patients, involving both sites examined though with different severity: osteopenia prevailed at femoral site, while osteoporosis was more prominent at lumbar spine (prevalently constituited by trabecular bone). Overall, the femoral site seemed to be more likely than the vertebral site influenced by biochemical and clinical factors. The only exception was pretransfusional $\mathrm{Hb}$, showing a positive correlation with vertebral mineralization; this could be explained by the high degree of ineffective erythropoiesis in the spine that can occurs in thalassemia syndromes. This finding is consistent with the interesting hypothesis that the chronic request for blood cell production can play a role in the etiology of osteoporosis, through overstimulation of the hematopoietic system increasing the number of osteoclasts and osteoblasts resulting in accelerated bone turnover [10].

In our analyses the statistical significance was reached more frequently by T-score, while Z-score seemed to be a less sensitive parameter. There is a statistical explanation to this behaviour: the relatively small number of cases can render the statistical power insufficient to detect smaller changes in Z-score, a parameter with high baseline variability. However, considering the very low prevalence of the disease, this is a sufficiently large cohort, and quite homogeneous as regards therapeutical approach and clinical management.

The statistically significant negative association between T-score and treatment with calcium and with bisphosphonates was expected, and compatible with the prescription of such drugs to the patients with more severe demineralization; the therapeutic effect of these medications could not be examined in our study, as the protocol did not include a longitudinal evaluation. Other parameters correlated to demineralization were gender (females are more involved), age and BMI, similarly to healthy individuals.

Even if we cannot address the issue of the prominent cause of bone disease in Thalassemia [11], the correlation between alkaline phosphatase and bone mineralization indicates a prevalent role of reduced deposition over increased reabsorption, as reported by other Authors [12], at variance with others [13, 14] . We could not confirm the same relationship with osteocalcin, another marker of osteoblastic activity [12, 15].

As far as the endocrine complications relevant to bone status are concerned, according to previous reports [16] in our study the hypogonadic patients of both genders were more affected from demineralization than the eugonadic, and, 
interestingly, the standard hormone replacement therapy (HRT) does not normalize bone condition. A possible

explanation may be the concurrence of factors interfering with mineralization, such as vitamin D deficiency, impairing the therapeutic effects of sex steroid hormones and also of bisphosphonates. A recent report [17] stated that an optimal vitamin D repletion is an essential pre-requisite for maximal efficacy of the anti-resorptive drugs in post-menopausal osteoporosis; a similar situation could be hypothesized in thalassemic population. In effect, low serum vitamin D levels have been reported in thalassemic populations; this was ascribed to impaired liver hydroxylation and intestinal absorption, with a causative role of iron overload suggested by a negative correlation between serum 25-OH-vitamin D and ferritin [18]. This correlation was not confirmed in our study $(\mathrm{p}=0.546)$. Unexpectedly, in our series the serum vitamin D was often low despite ongoing treatment with metabolites, compatible with a defect in absorption or metabolism [18] but also with low compliance to the therapy; the latter is quite common using associations with calcium salts, badly tolerated. Anyway, the contribution of vitamin D to bone status is probably more complex than presently recognized, and neither vitamin D concentrations nor ongoing treatment did correlate with mineralization status in our and earlier reports [19]. Conversely, impairment of parathyroid function was inversely related to mineralization status, indicating a protective effect on bone of this endocrine complication [19, 20]. In our study the relationship was more evident at the femoral level than at lumbar spine; this was unexpected, as chronic PTHdeprivation should influence more strongly the sites where trabecular content is maximum. Probably, in this peculiar population the superimposed pathologic mechanisms concurring to bone disease can produce net effects difficult to predict in the long-term.

Finally, an important consideration emerges from our statistical analysis: the model utilized could account for the $29.4 \%$ of T-score variance at the femur and the $32.03 \%$ at the spine. This indicates that the variables significant in the univariate analysis could not explain satisfactorily the mineralization defect. Again, this may be ascribed to insufficient statistical power, linked to the restricted number of cases in a monocentric study, or alternatively to the influence of pathogenetically involved factors beyond those included in our analysis. The indices studied reflect bone marrow expansion, iron overload, endocrine function and bone metabolism, but further physiological, pathological, genetic and iatrogenic factors can work in this multifactorial disease.

Furthermore, early preventive intervention and management are needed to influence bone health in adulthood, as demonstrated by normal Z-scores found in children and young adolescents with Thalassemia Major by Christoforidis et al [21]. Our patients are probably comparable for the quality of clinical management; however the different results emerged from our study suggest that transfusion and chelation treatment can prevent bone demineralization only when applied early from childhood. 
A deeper learning of the molecular mechanisms causing demineralization, as well as the optimal prevention and

1

2

3

4

5

6

7

8

9

10

11

12

13

14

15

16

17

18

19

20

21

22

23

24

25

26

27

28

29

30

31

32

33

34

35

36

37

38

39

40

41

42

43

44

45

46

47

48

49

50

51

52

53

54

55

56

57

58

59

60

61

62

63

64

65

treatment of thalassemia from infancy, could perhaps allow to avoid complications and further ameliorate the quality of life in these patients. 
Fig 1 Bone mineralization state (normal / osteopenia / osteoporosis) of the Thalassemic patients as distributed on the basis of site and gender, according to the WHO osteoporosis classification. Osteopenia was the prominent finding at the femur, while at lumbar spine osteoporosis was more prevalent $(\mathrm{p}<0.001)$. The same distribution was confirmed when the patients were separately considered according to gender, being more evident in the male group

Table 1 Clinical characteristics of the patients by gender at the time when mineralization was studied

Table 2 Biochemical and hormonal indices related to bone metabolism in 111 patients affected by Thalassemia Major

Table 3 Statistic results (mean \pm standard deviation and $p$ value) of comparisons between clinical/biochemical dicotomic variables and $\mathrm{T}$ or $\mathrm{Z}$ - scores at femoral and vertebral sites

Table 4 Correlation analysis (Pearson's correlation indices and two-tailed $p$ values) between bone mineralization (expressed by $\mathrm{T}$ and Z-scores) and clinical/biochemical parametric variables 


\section{References}

1. Borgna-Pignatti C, Cappellini MD, De Stefano P, Del Vecchio GC, Forni GL, Gamberini MR, Ghilardi R, Origa R, Piga A, Romeo MA, Zhao H, Cnaan A (2005) Survival and complications in thalassemia. Ann N Y Acad Sci 1054:4047

2. Vogiatzi MG, Macklin EA, Fung EB, Vichinsky E, Olivieri N, Kwiatkowski J, Cohen A, Neufeld E, Giardina PJ (2006) Prevalence of fractures among the thalassemia syndromes in north america. Bone 38(4):571-575

3. Vogiatzi MG, Macklin EA, Fung EB, Cheung AM, Vichinsky E, Olivieri N, Kirby M, Kwiatkowski JL, Cunningham M, Holm IA, Lane J, Schneider R, Fleisher M, Grady RW, Peterson CC, Giardina PJ, Thalassemia Clinical Research Network (2009) Bone disease in thalassemia: A frequent and still unresolved problem. J Bone Miner Res 24(3):543-557

4. Cappellini M.D, Cohen A, Eleftheriou A, Piga A, Porter J, Taher A (2008) Guidelines for the clinical management of thalassemia; $2^{\text {nd }}$ revised Edition. Thalassemia International Federation. http://thalassemia.org.cy

5.Wood JC, Enriquez C, Ghugre N, Tyzka JM, Carson S, Nelson MD, Coates TD (2005) MRI R2 and R2* mapping accurately estimates hepatic iron concentration in transfusion-dependent thalassemia and sickle cell disease patients. Blood 106 (4):1460-65

6. Dubey AP, Parakh A, Dublish S (2008) Current trends in the management of beta thalassemia. Indian J Pediatr 75(7):739-743

7. Perrotta S, Cappellini MD, Bertoldo F, Servedio V, Iolascon G, D'Agruma L, Gasparini P, Siciliani MC, Iolascon A (2000) Osteoporosis in beta-thalassaemia major patients: Analysis of the genetic background. Br J Haematol 111(2):461-466

8. Origa R, Fiumana E, Gamberini MR, Armari S, Mottes M, Sangalli A, Paglietti E, Galanello R, Borgna-Pignatti C (2005) Osteoporosis in beta-thalassemia: Clinical and genetic aspects. Ann N Y Acad Sci 1054:451-456

9. Skordis N (2009) The labyrinth of bone disease in thalassaemia: The search for ariadne's thread continues. Eur J Haematol 82(1):13-14 
10. Gurevitch O, Slavin S (2006) The hematological etiology of osteoporosis. Med Hypotheses 67(4):729-735

11. Voskaridou E, Terpos E (2008) Pathogenesis and management of osteoporosis in thalassemia. Pediatr Endocrinol Rev 6 Suppl 1:86-93

12. Salama OS, Al-Tonbary YA, Shahin RA, Eldeen OA (2006) Unbalanced bone turnover in children with betathalassemia. Hematology 11(3):197-202

13. Dresner Pollack R, Rachmilewitz E, Blumenfeld A, Idelson M, Goldfarb AW (2000) Bone mineral metabolism in adults with beta-thalassaemia major and intermedia. Br J Haematol 111(3):902-907

14. Voskaridou E, Kyrtsonis MC, Terpos E, Skordili M, Theodoropoulos I, Bergele A, Diamanti E, Kalovidouris A, Loutradi A, Loukopoulos D (2001) Bone resorption is increased in young adults with thalassaemia major. Br J Haematol 112(1):36-41

15. Morabito N, Gaudio A, Lasco A, Atteritano M, Pizzoleo MA, Cincotta M, La Rosa M, Guarino R, Meo A, Frisina N (2004) Osteoprotegerin and RANKL in the pathogenesis of thalassemia-induced osteoporosis: New pieces of the puzzle. J Bone Miner Res 19(5):722-727

16. Carmina E, Di Fede G, Napoli N, Renda G, Vitale G, Lo Pinto C, Bruno D, Malizia R, Rini GB (2004) Hypogonadism and hormone replacement therapy on bone mass of adult women with thalassemia major. Calcif Tissue Int 74(1):68-71

17. Adami S, Giannini S, Bianchi G, Sinigaglia L, Di Munno O, Fiore CE, Minisola S, Rossini M (2009) Vitamin D status and response to treatment in post-menopausal osteoporosis. Osteoporos Int 20(2):239-244

18. Napoli N, Carmina E, Bucchieri S, Sferrazza C, Rini GB, Di Fede G (2006) Low serum levels of 25-hydroxy vitamin D in adults affected by thalassemia major or intermedia. Bone 38(6):888-892

19. Angelopoulos NG, Goula A, Rombopoulos G, Kaltzidou V, Katounda E, Kaltsas D, Tolis G (2006)

Hypoparathyroidism in transfusion-dependent patients with beta-thalassemia. J Bone Miner Metab 24(2):138-145

20. Laway BA, Goswami R, Singh N, Gupta N, Seith A (2006) Pattern of bone mineral density in patients with sporadic idiopathic hypoparathyroidism. Clin Endocrinol (Oxf) 64(4):405-409 
21. Christoforidis A, Kazantzidou E, Tsatra I, Tsantali H, Koliakos G, Hatzipantelis E, Katzos G, Athanassiou-Metaxa

1

2

3

4

5

6

7

8

9

10

11

12

13

14

15

16

17

18

19

20

21

22

23

24

25

26

27

28

29

30

31

32

33

34

35

36

37

38

39

40

41

42

43

44

45

46

47

48

49

50

51

52

53

54

55

56

57

58

59

60

61

62

63

64

65 M (2007) Normal lumbar bone mineral density in optimally treated children and young adolescents with betathalassaemia major. Hormones (Athens) 6(4):334-340 
